\title{
Parametric amplification of topological interface states in synthetic Andreev bands
}

\author{
I. Septembre, ${ }^{1}$ S. Koniakhin,,${ }^{1,2}$ J. S. Meyer, ${ }^{3}$ D. D. Solnyshkov, ${ }^{1,4}$ and G. Malpuech ${ }^{1}$ \\ ${ }^{1}$ Institut Pascal, PHOTON-N2, Université Clermont Auvergne, CNRS, SIGMA Clermont, F-63000 Clermont-Ferrand, France \\ ${ }^{2}$ St. Petersburg Academic University-Nanotechnology Research and Education Centre of the Russian Academy of Sciences, \\ 194021 St. Petersburg, Russia \\ ${ }^{3}$ Université Grenoble Alpes, CEA, INP Grenoble, IRIG, PHELIQS, 38000 Grenoble, France \\ ${ }^{4}$ Institut Universitaire de France (IUF), 75231 Paris, France
}

(Received 10 December 2020; accepted 12 May 2021; published 2 June 2021)

\begin{abstract}
A driven-dissipative nonlinear photonic system (e.g., exciton-polaritons) can operate in a gapped superfluid regime. We demonstrate theoretically that the reflection of a linear wave on this superfluid is an analog of the Andreev reflection of an electron on a superconductor. A normal region surrounded by two superfluids is found to host Andreev-like bound states. These bound states form topological synthetic bands versus the phase difference between the two superfluids. Changing the width of the normal region allows us to invert the band topology and to create "interface" states. Instead of demonstrating a linear crossing, synthetic bands are attracted by the nonlinear non-Hermitian coupling of bosonic systems, which gives rise to a self-amplified strongly occupied topological state.
\end{abstract}

DOI: 10.1103/PhysRevB.103.214504

Topological physics relies on the specific structure of the eigenstates of Hamiltonians in a parameter space. It has become one of the most active fields of research of the past few decades. Topological invariants were successively used to characterize superfluid excitations (quantum vortices) [1,2], solitons in polyacetylene [3], Landau levels [4], electronic Bloch bands in solids [5], and more generally energy bands in periodic media [6]. A related concept is the bulk-boundary correspondence $[7,8]$, which associates the change of the band topology with a gap closing and therefore with the existence of an interface state between media of different topologies. Such interface states demonstrate unique properties, like the one-way transport in topological insulators [9].

The recent proposals and implementations of topological media supporting protected edge states in nonlinear systems opened new possibilities [10-18]. The most well-known examples are topological lasers, where lasing occurs in the topological edge states of a photonic lattice [19-22]. Another recent orientation is synthetic topological matter, where the parameters of the Hamiltonian are not imposed by the system itself (such as the wave vector in Bloch bands), but rather externally by experimental conditions. This approach allows one to explore inaccessible regimes, such as the four-dimensional (4D) quantum Hall effect [23]. One implementation of synthetic topological matter relies on using Josephson junctions between conventional superconductors [24]. The dependence of Andreev bound states versus the phase difference between the superconductors forming the junction allows one to define synthetic bands with the synthetic dimensionality controlled by the number of terminals. These bands were found to be topological, showing Weyl singularities, and significant efforts are being made to implement them [25,26]. Andreev reflection occurs at the interface of a superconductor [27], where an incoming electron undergoes an anomalous reflection, becoming a hole excitation with reversed wave vector, charge, and mass. The analogy between Andreev reflection and the reflection of a wave over a Bose-Einstein condensate has been studied theoretically [28]. In photonics, one can implement a superconductor analog using resonant driving, typical for cavity exciton-polaritons [29,30]. In such a case, a gap opens in the spectrum of excitations of the pumped modes, which corresponds to the formation of a "gapped superfluid." It is possible to spatially modulate the pump intensity to realize two or more gapped regions with controllable phase, separated by normal (nonsuperfluid) regions [31-33].

In this work, we study the analog of the 1D Andreev reflection on a gapped driven-dissipative polariton superfluid. We show the existence of Andreev-like bound states between two driven superfluids. The topology of the synthetic bands defined versus the phase difference between the superfluids is nontrivial. The interface states between the regions of inverted topology are found to be self-amplified. They are macroscopically occupied topologically protected Andreev-like states.

\section{THE MODEL}

We consider a 1D strongly coupled microcavity [29,30,34]. The 1D superfluid wave function $\psi$ is described by the drivendissipative Gross-Pitaevskii (GP) equation for scalar particles [29], analogous to the equation describing the electric field amplitude in a nonlinear system [35]:

$$
i \hbar \frac{\partial \psi}{\partial t}=\left[-\frac{\hbar^{2}}{2 m} \frac{\partial^{2}}{\partial x^{2}}-i \gamma+\alpha|\psi|^{2}\right] \psi+P .
$$

$P=e^{-i \omega_{p} t}$ is the quasiresonant pumping term at normal incidence, $\alpha>0$ is the repulsive interaction constant, and $\gamma$ is the decay rate. The bare mode dispersion is quasiparabolic (mass $m$ ), typical for photons or exciton-polaritons in a microcavity. 
The cavity is pumped through the mirrors at normal incidence (in the $z$ direction), and the in-plane propagation occurs in the $x$ direction. The polariton density $|\psi(x, t)|^{2}$ determines the intensity of the light emitted by the cavity, which can be detected from the outside by optical means. Polarization effects are neglected. The spatially homogeneous solution $\psi_{s} e^{-i \omega_{p} t}$ shows a bistable behavior [36] with a large occupation above the bistable threshold. To simplify the analytics, we consider the limits $\gamma \rightarrow 0$ and $T=0 \mathrm{~K}$, which is appropriate for low-temperature experiments $[29,30,34]$. The wave function describing weak superfluid excitations ("bogolons") reads

$$
\psi(x, t)=e^{-i \omega_{p} t}\left(\psi_{s}+u e^{i k x} e^{-i \omega t}+v^{*} e^{-i k x} e^{i \omega^{*} t}\right) .
$$

Here, one plane-wave excitation is coupled to its complex conjugate. Inserting this wave function into Eq. (1) yields the Bogoliubov-de Gennes (BdG) equations:

$$
\left(\begin{array}{cc}
\mathcal{L} & \alpha \psi_{s}^{2} \\
-\alpha \psi_{s}^{* 2} & -\mathcal{L}^{*}
\end{array}\right)\left(\begin{array}{l}
u \\
v
\end{array}\right)=E\left(\begin{array}{l}
u \\
v
\end{array}\right),
$$

where $\mathcal{L}=\left(\epsilon_{k}-E_{p}+2 \alpha n\right) ; u, v$ are the Bogoliubov coefficients, $\epsilon_{k}=\hbar^{2} k^{2} / 2 m$, and $\psi_{s}=\sqrt{n} e^{i \phi}$. The energy $E=\hbar \omega$, measured with respect to the laser $E_{p}=\hbar \omega_{p}$, is found by canceling the determinant, and it reads

$$
E^{2}=\left(\epsilon_{k}+\alpha n-E_{p}\right)\left(\epsilon_{k}+3 \alpha n-E_{p}\right) .
$$

A bogolon is formed by the superposition of two plane waves of amplitude $u$ and $v^{*}$ at energies $E,-E$. With $E$ positive, the ratio $|u| /|v|$ is fixed by Eq. (3) and is larger than 1 . The specific choice of the normalization condition $|u|^{2}-|v|^{2}=1$ defines a bogolon as a particle of a positive total energy $\left(|u|^{2}-|v|^{2}\right) E$, but containing amplitudes at both $E$ and $-E$. When $\alpha n>E_{p}$, the energy spectrum (containing both positive and negative parts) shows a gap $2 \Delta$ centered at the pump energy:

$$
\Delta=\sqrt{\left(\alpha n-E_{p}\right)\left(3 \alpha n-E_{p}\right)} .
$$

\section{ANDREEV REFLECTION ANALOG}

We consider two semi-infinite regions [Fig. 1(a)]. The normal (left) part is not pumped and is described by the 1D Schrödinger equation. The superfluid (right) part is resonantly pumped and is described by the 1D GP equation (1). We consider an incident wave, created by a weak probe laser and coming from the $\mathrm{N}$-region toward the superfluid at an energy within the gap. The in-plane wave vector of the probe is controlled by the angle of incidence of the probe laser $\theta$ as $k_{x}=\omega \sin \theta / c$. We therefore consider evanescent bogolons:

$$
\psi(x, t)=e^{-i \omega_{p} t}\left(\psi_{s}+u e^{-\kappa x} e^{-i \omega t}+v^{*} e^{-\kappa x} e^{+i \omega^{*} t}\right) .
$$

The BdG equations read as Eq. (3), but with $\mathcal{L}=$ $\left(-\epsilon_{\kappa}-E_{p}+2 \alpha n\right)$ and $\epsilon_{k}=\hbar^{2} \kappa^{2} / 2 m$. Because of the nonHermiticity of the BdG equations, the characteristic equation yields purely evanescent modes with an inverse decay length:

$$
\kappa_{ \pm}=\sqrt{2 m\left(2 \alpha n-E_{p} \pm \sqrt{(\alpha n)^{2}+E^{2}}\right)} / \hbar .
$$

Here, the solutions are not propagative-decaying modes as in the fermionic case [37], but purely evanescent. There exist two evanescent waves at a given positive $E$, associated with the

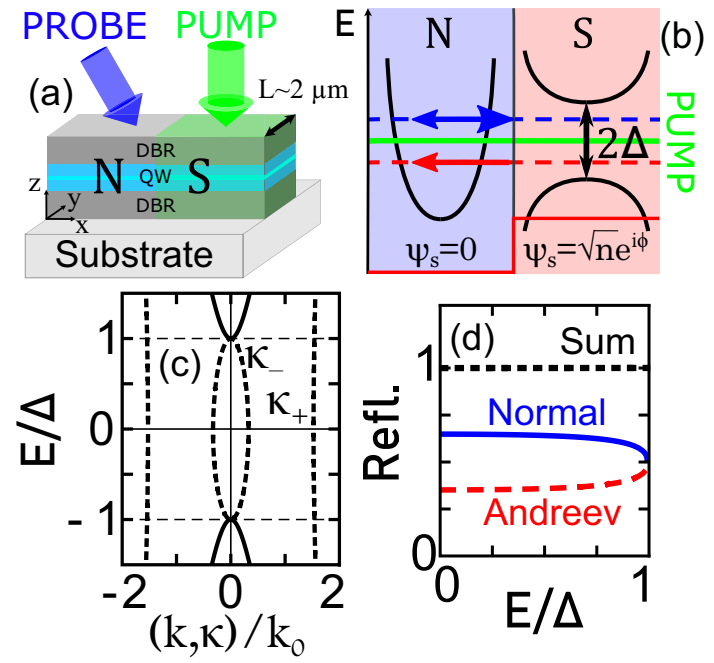

FIG. 1. (a) Wire microcavity based on distributed Bragg reflectors (DBRs) with embedded quantum wells (QWs). The S-region is pumped by a laser under normal incidence, while the N-region is not. A weak probe laser creates a wave in the N-region moving toward the superfluid. (b) Schematic energy bands. Black: energy dispersion of propagative modes. Green: pump laser energy. Blue: incident probe and normal reflection. Red: Andreev reflection. (c) Bogolon energy versus the wave vector $k$ (propagative states, solid lines) and vs the inverse decay lengths $\kappa_{ \pm}$(evanescent states, dashed lines), discussed in Appendix A. $k_{0}=\left.k\right|_{E=0}=\sqrt{2 m E_{p}} / \hbar$. (d) Normal and Andreev reflection coefficients vs energy.

two different decay lengths:

$$
\begin{aligned}
& u_{ \pm}=\frac{\sqrt{\sqrt{(\alpha n)^{2}+E^{2}} \mp E} e^{i \phi},}{\sqrt{2 E}} \\
& v_{ \pm}= \pm \frac{\sqrt{\sqrt{(\alpha n)^{2}+E^{2}} \pm E}}{\sqrt{2 E}} e^{-i \phi} .
\end{aligned}
$$

The "minus" state has a longer decay length and $\left|u_{-}\right|>$ $\left|v_{-}\right|$. Its dominant component $u_{-}$has an energy $E^{\prime}=E>0$ (the minority component $v_{-}$has $E^{\prime \prime}<0$ ) and the state is normalized as $\left|u_{-}\right|^{2}-\left|v_{-}\right|^{2}=1$. It is quite similar to propagative bogolons, continuing their dispersion within the gap [Fig. 1(c)]. We call this type of state, where the positive energy component dominates, a "particle." The "plus" state has a shorter decay length and $\left|u_{+}\right|<\left|v_{+}\right|$. Its dominant component has a negative energy $E^{\prime}=-E<0$ and $\left|u_{+}\right|^{2}-\left|v_{+}\right|^{2}=-1$. This evanescent solution has no propagative counterpart. The $\kappa_{+}$branch shown in Fig. 1(c) is disconnected from the propagative states dispersion and can be assimilated to "hole" states. They are associated with a local decrease of the particle density with respect to the homogeneous superfluid.

We now consider a plane wave of energy $-\Delta<E<\Delta$ created by a probe laser in the N-region [Fig. 1(b)] and propagating toward the superfluid. It excites the two abovementioned types of evanescent bogolons, provoking reflection at both $E$ and $-E$. The $\mathrm{N}$-region wave function reads

$$
\psi_{N}=\left(\begin{array}{l}
A e^{i k_{1} x}+B e^{-i k_{1} x} \\
C e^{i k_{2} x}+D e^{-i k_{2} x}
\end{array}\right),
$$




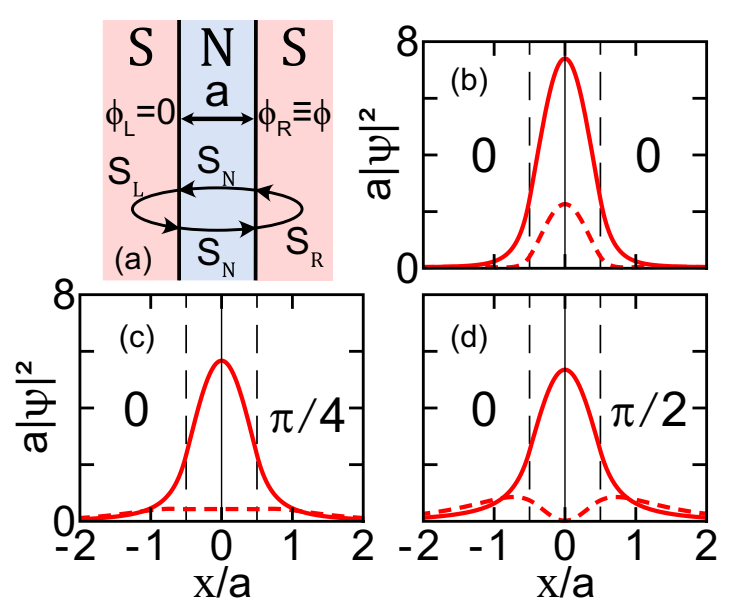

FIG. 2. (a) Schematic representation of a bosonic SNS junction with its principal characteristics. The matrices depicting each scattering event are also represented. (b)-(d) Probability density (solid line: majority component; dashed line: bogolon image) for $a=2.6 \mu \mathrm{m}$ and a phase difference of (b) 0 , (c) $\pi / 4$, and (d) $\pi / 2$.

with $k_{1,2}=\sqrt{2 m\left(E_{p} \pm E\right)} / \hbar$, valid for $\Delta<E_{p}$ (Appendix B considers evanescent states in the $\mathrm{N}$-region). The superfluid wave function combines two evanescent waves:

$$
\psi^{S}=\eta_{+}\left(\begin{array}{c}
u_{+} \\
v_{+}
\end{array}\right) e^{-\kappa_{+} x}+\eta_{-}\left(\begin{array}{c}
u_{-} \\
v_{-}
\end{array}\right) e^{-\kappa_{-} x} .
$$

To compute the reflection coefficients, we take $A=1 / \sqrt{v_{1}}$, $C=0$. In that case, $B=r_{N p} / \sqrt{v_{1}}$ and $D=r_{A p} / \sqrt{v_{2}}$ are the normal and Andreev reflection coefficients for an incident "particle," respectively (dominant positive energy component $E^{\prime}>0$ ). The group velocities allow us to conserve the current. Similarly, $A=0, C=1 / \sqrt{v_{2}}$ corresponds to $B=$ $r_{A h} / \sqrt{v_{1}}, D=r_{N h} / \sqrt{v_{2}}$, the reflection coefficients for an incident "hole" having a dominant negative energy component $E^{\prime}<0$. The continuity of the wave functions and of their derivatives at the interface gives an analytical expression for these reflection coefficients (see Appendix C). They are plotted in Fig. 1(d) for $m=5 \times 10^{-5} m_{0}, E_{p}=0.5 \mathrm{meV}$, and $\alpha n \approx 1.11 E_{p}$, characteristic for GaAs-based microcavities. This yields a gap $\Delta \approx 0.5 E_{p}$. The Andreev-like reflection is comparable in amplitude with the normal reflection, and the reflection is total $\left(\left|r_{N p, h}\right|^{2}+\left|r_{A p, h}\right|^{2}=1\right)$. Here, the superfluid gap really acts as a potential barrier, providing finite backscattering as in electronic Josephson junctions with an oxide barrier [37]. Ultimately, this phenomenon can also be interpreted $[38,39]$ as a nonlinear frequency conversion (optical phase conjugation), studied in the 1970s in nonlinear optics [40].

\section{ANDREEV BOUND STATES ANALOG}

We next consider a superfluid-normal-superfluid (SNS) junction [Fig. 2(a)]. The superfluids have different phases, $\phi_{L}=0$ and $\phi_{R} \equiv \phi$, fully controlled by the phases of the pumping lasers. The width of the N-region is $a$. In the absence of Andreev reflection, this structure is equivalent to a potential well (the superfluids forming the barriers) and shows quantized eigenstates $E_{n}^{\prime}$, labeled by integer quantum numbers $n$. Andreev reflection provides a correction to these quantized states and generates a second energy component $E_{n}^{\prime \prime}=-E_{n}^{\prime}$. We call this second component a "bogolon image," by analogy with the well-known concept of "phonon replica." Indeed, this second frequency appears because of the properties of the excitations of the system, which are bogolons, but it is not an exact replica, as we will see below, so the word "image" is more relevant.

The Andreev bound states are found using the scattering matrix formalism. The matrix describing the reflections at the interfaces reads

$$
S_{R(L)}=\left(\begin{array}{cc}
r_{N p}^{R(L)} & r_{A h}^{R(L)} \\
r_{A p}^{R(L)} & r_{N h}^{R(L)}
\end{array}\right) .
$$

The total scattering matrix reads $S_{T}=S_{L} S_{N} S_{R} S_{N}$ [see Fig. 2(a)], where $S_{N}$ describes the propagation in the N-region (see Appendix C). A bound eigenstate exists if $\pm E$ satisfy [41]

$$
\operatorname{det}\left[\mathbb{I}-S_{T}\right]=0 .
$$

Depending on parameters, the eigenenergies can be either real (stationary bound states) or, due to non-Hermiticity of the BdG matrix, complex (self-amplified bound states). The eigenvectors of $S_{T}$ determine the wave function via Eqs. (9) and (10) and whether a state is either particlelike $\left(E^{\prime}>0\right)$ or holelike $\left(E^{\prime}<0\right)$. Figures 2(b)-2(d) show an example of a holelike bound state $(n=1)$. In Fig. 2(b), $\phi=0$ and both energy components are $s$-like. In Fig. 2(d), $\phi=\pi / 2$. The main component keeps its parity, whereas the bogolon image becomes $p$-like, because of the phase shift. Interestingly, the probability current $J_{ \pm}$associated with each energy component $+E,-E$ in the N-region (see Appendix D for details) takes a form similar to the Josephson current in superconducting junctions:

$$
J_{ \pm} \approx \pm J_{0} \sin 2 \phi .
$$

The total current $J=J_{+}+J_{-}$is zero.

\section{TOPOLOGICAL SYNTHETIC BANDS}

Both positive and negative energy components of a bound state form synthetic energy bands [42], where the phase $\phi$ plays the role of the wave vector. For a given band, the wave function is a superposition of two counterpropagating plane waves, as defined by Eq. (9). As for Bloch bands in solids, the amplitudes of these two plane waves define a pseudospinor $X^{+} \sim(A, B)^{T}$ for positive energies and $X^{-} \sim(C, D)^{T}$ for negative energies.

The pseudospinor evolution along the band allows us to compute the Zak phase $[43,44]$ :

$$
\Phi_{\mathrm{Zak}}^{ \pm}=\int\left\langle X^{ \pm} \mid i \frac{\partial X^{ \pm}}{\partial \phi}\right\rangle d \phi .
$$

The dissipative character of the system is by itself not an obstacle to the computation of topological quantities and to the use of the bulk-boundary correspondence, provided that the bands, broadened by dissipation, remain well separated [45].

Figures 3(a)-3(c) show the synthetic bands and their Zak phases for three thicknesses $a$. In Figs. 3(a) and 3(c) all energies are real. The bands shown in blue are particlelike 

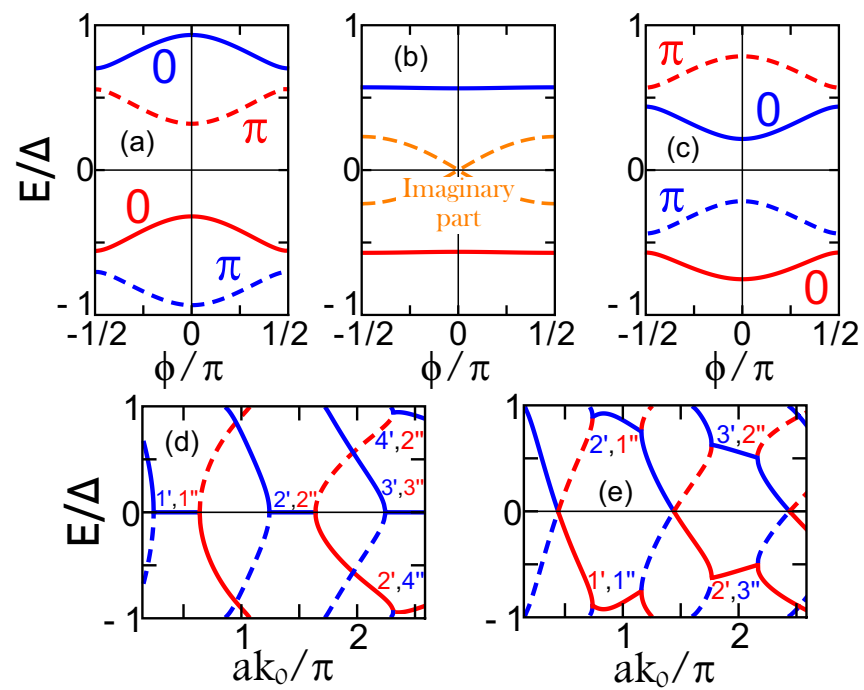

FIG. 3. Synthetic band energies vs $\phi$ (a)-(c) and $a$ (d), (e). Blue lines: particlelike states. Red lines: holelike states; the dominant (minority) component is shown as a solid (dashed) line. (a) $a=6.8 \mu \mathrm{m}$; $0, \pi$-Zak phases. (b) $a=7.6 \mu \mathrm{m}$; dashed lines: imaginary part of energy. (c) $a=8.4 \mu \mathrm{m}$; $0, \pi$-Zak phases. (d) $\phi=0$. (e) $\phi=\pi / 2$. (d), (e) The numbers indicate the quantum number of original trapped states from which stem the crossing bands.

states with a dominant positive energy part $\left(E^{\prime}>0\right.$, solid lines). Their negative energy counterpart $\left(E^{\prime \prime}<0\right.$, bogolon image) is smaller in amplitude (dashed lines) and shows a nonzero Zak phase, which translates the change of the wave-function symmetry versus $\phi$ illustrated in Fig. 2. The corresponding nontrivial band topology can also be understood in terms of the motion of the pseudospinors $X^{+}, X^{-}$ on a Bloch sphere versus $\phi$. Indeed, at $\phi=0, C^{\prime}= \pm D^{\prime}$. The current is zero, and the associated pseudospin lies in the plane of a Bloch sphere representation. At $\phi \approx \pi / 4, D^{\prime}=0$. The current at each energy is maximal, and the pseudospin points toward the pole. Finally, at $\phi=\pi / 2, C^{\prime}=\mp D^{\prime}$ : the symmetry of the state has changed. Between $\phi=-\pi / 2$ and $\pi / 2$, the pseudospin covers a full great circle of the Bloch sphere, constrained by the mirror symmetry of the problem as shown in Appendix E. The accumulated Zak phase is $\pi$. On the other hand, the pseudospin of the majority component moves slightly toward the pole at $\phi \approx \pi / 4$ to go back to its original position at $\phi=\pi / 2$. In general, the band associated with the dominant energy component of the bogolon $E^{\prime}$ shows a zero Zak phase, whereas the minority component $E^{\prime \prime}$ is topologically nontrivial.

The band topology is inverted between Figs. 3(a) and 3(c), which are computed for different $a$ values. This implies a gap closing for a critical thickness $a_{0}$. This topological band crossing in parameter space is reminiscent of a topological phase transition in HgTe QWs versus the well thickness [46]. Such band inversion gives rise to Jackiw-Rebbi states [47] in 2D and Su-Schrieffer-Heeger solitons [3] in 1D. It is at the heart of topologically protected edge or interface states through the bulk-boundary correspondence.

In our bosonic system of interacting particles, crossing bands interact through a non-Hermitian coupling [29] [Eq. (3)]. Hamiltonians with non-Hermitian perturbations are known to exhibit complex eigenvalues close to the degeneracy points of the original unperturbed Hermitian Hamiltonians $[48,49]$. A degeneracy point (diabolical point) is thus transformed into a pair of exceptional points, between which the eigenvalues are complex (and conjugate). This also happens in our system. Figure 3(b) shows the synthetic bands at the critical thickness $a_{0}$. Instead of simply crossing, the bands merge and opposite imaginary parts appear, meaning that one of the states gets amplified. This amplification occurs when a bogolon image is resonant in energy and overlaps (this is called a "phase-matching condition" for parametric amplification [50]) with the linear eigenstate of the potential trap formed by the superfluid gaps. Figures 3(d) and 3(e) show for $\phi=0$ and $\phi=\pi / 2$, respectively, the mode energy versus $a$. For $\phi=0$, amplification occurs when the majority and minority component have the same parity $E_{n}^{\prime}=E_{m}^{\prime \prime}(m-n=2 i$, $i \in \mathcal{Z}$ ), which occurs, for example, when the potential well states $n=1,2, \ldots$ are resonant with the laser. For $\phi=\pi / 2$, the amplification occurs when the bogolon image of a state of given parity becomes resonant with an original trapped state of different parity $E_{n}^{\prime}=E_{m}^{\prime \prime}(m-n=2 i+1, i \in \mathcal{Z})$. These conditions can also be considered as a particular example of selection rules for the transitions from the pumped mode to the bogolon modes due to nonlinear interactions.

To summarize, when the bands are well-separated, the topological invariants are well-defined in spite of the nonHermiticity of the system [45]. Since the band topology is inverted for $a>a_{0}$ and $a<a_{0}$, the bands must cross in between the two limits. This crossing provides a degeneracy, which gives rise to complex eigenvalues and mode amplification [49] thanks to the non-Hermiticity. We note that when the bands are crossing each other, the topological invariants for each of them cannot be calculated anymore, independently of the Hermiticity of the system.

In conservative quantum fluids, such as atomic BoseEinstein condensates, a positive imaginary part of the energy of weak excitations is considered as a signature of an instability. Indeed, the growth of such modes occurs at the expense of the condensate, and moreover, these modes with positive imaginary parts usually form a continuum. When their population becomes macroscopic, it means the destruction of the condensate, because of a strong decrease of its population. In our case, there are two important qualitative differences. First of all, the modes with positive imaginary parts do not form a continuum: their number is finite. Even in a conservative system, this configuration does not lead to an instability, but to periodic oscillations of the population between the initially populated state and the two other states with energy conservation. Even if the population of the initial state drops to zero, it does not mean that the condensate is destroyed, because the coherence of the whole system is conserved, and the initial state is then repopulated from the two other states. This is the fundamental difference between the coupling to a continuum and the coupling to a countable (even infinite) number of modes, as shown by the revival phenomenon in quantum optics [51,52]. Second, the system is a driven-dissipative one, with a continuous pump, constantly replenishing the original state with macroscopic population. A constant and comparable population is established in all three states. In optics, such an effect is called optical parametric oscillations [53-55]. 
Together with optical parametric amplification [50,56], it has important practical applications, including the generation of entangled photon pairs [57]. While the initial growth of the amplified modes (called "signal" and "idler" in optics) is indeed exponential, the saturation occurs due to the pump depletion, and the behavior of the modes in this case is described by the elliptic sine function [56]. In our particular case, the amplified modes are localized in the $\mathrm{N}$-region, and the amplifying nonlinearity is taking place at the superfluid interfaces. These modes cannot propagate within the gap of the superfluid. So, their growth does not lead to the instability of the semi-infinite superfluids, as confirmed by numerical simulations. Our predictions are valid for all optical systems with nonlinearities (waveguides [58], atomic vapors [59], photorefractive crystals [60]), because in all of them a confinement in two dimensions (to a 1D propagative channel) can be organized.

\section{NUMERICAL SIMULATIONS}

The analytical solution shown in the previous sections applies to a $1 \mathrm{D}$ system. We have demonstrated that it follows from the topological reasoning that for a certain width of the $\mathrm{N}$-region, the two modes in this region get amplified. While studying a full 2D problem analytically is complicated and goes beyond the scope of the present work, it is natural to check if the main conclusions could be verified in a 2D system with a varying width of the N-region. In addition to the modes with zero $k_{y}$ found above, the system also contains multiple modes with nonzero $k_{y}$. However, the conclusions for the modes with $k_{y}=0$ still hold, because they are unaffected by the presence of the other modes, and they still must cross each other for a particular critical width because of the topology inversion. The extra modes can be seen as additional losses, which do not change the situation qualitatively, since the system nevertheless exhibits losses through the cavity mirrors. It means that the width of the N-region should not vary too rapidly, and the disorder should be sufficiently low, otherwise the total losses might overcome the gain.

To show this, we numerically solve the 2D GrossPitaevskii equation over time with a weak probe exciting the bogolon states, and a finite lifetime $\tau=\hbar / 2 \gamma=30$ ps. The width $a$ varies versus $y$ as $a(y)=a_{0}+y / l$, where $l=64 \mu \mathrm{m}$. The calculated spectra are presented in Fig. 4 for $a<a_{0}$ for $\phi=0$ (a) and $\phi=\pi / 2$ (b). The two energy components of each of the Andreev states are marked in the figure (e.g., $1^{\prime}$ is the "original" trapped state and 1 " is its bogolon image). The symmetries of the bogolon images $1^{\prime \prime}$ and $2^{\prime \prime}$ clearly change. The gap closes at $a=a_{0}$, where each original state is resonant with the bogolon image of the other: $E_{2}^{\prime}=E_{1}^{\prime \prime}$ and $E_{1}^{\prime}=E_{2}^{\prime \prime}$, and their symmetries also coincide for $\phi=\pi / 2$. As a result, the Andreev states are amplified and dominate the spectrum [Fig. 4(d)]. Figure 4(c) shows the spatial distribution of emission, with the amplified Andreev state visible at $y=0 . \gamma$ should be 5-10 times smaller than the gap for the amplification to be experimentally observable, which is clearly realistic. We stress that the N-region is a noninteracting medium, otherwise solitons form [31-33], and the approximations used become invalid.

To summarize, we predict an Andreev reflection analog in a photonic driven-dissipative gapped superfluid. Such sys-
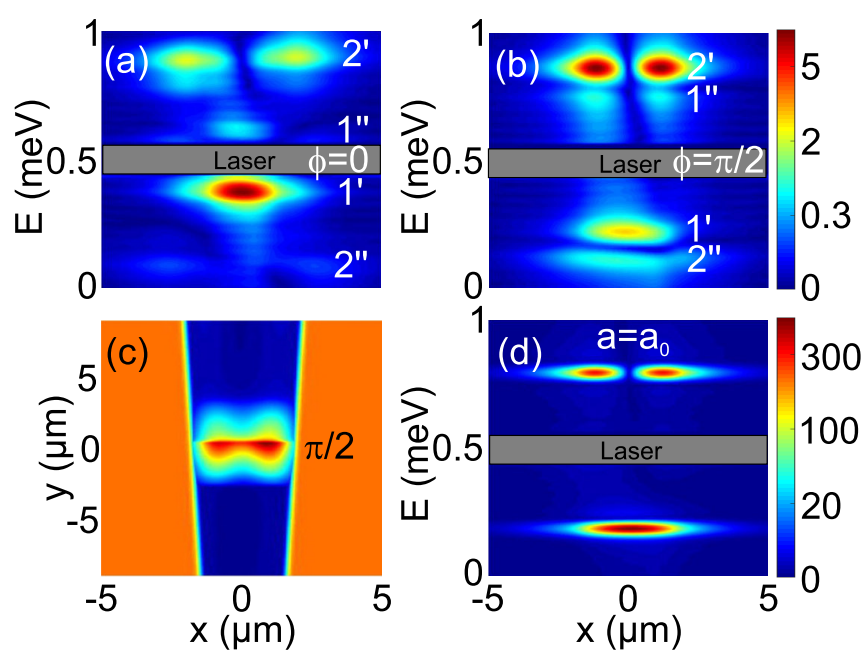

FIG. 4. Numerically calculated spectrum of the Andreev states. Two energy components are visible for each of the two states (marked $1^{\prime}, 1^{\prime \prime}$ and $2^{\prime}, 2^{\prime \prime}$ ) for $a<a_{0}$ : (a) $\phi=0$, (b) $\phi=\pi / 2$. (c) Numerically calculated emission intensity for a system with varying width $a$. The amplified states are visible at $y=0$. (d) The spectrum of the resonantly amplified states at $y=0$. The laser energy is cut out for all images. False color shows the polariton density $|\psi|^{2}$.

tems can be used to form bosonic SNS junctions hosting Andreev-like bound states. These bound states form topologically nontrivial synthetic bands versus the phase difference between the pumping lasers. By changing the width of the $\mathrm{N}$-region, one can invert their topology, and the associated topologically protected interface states get self-amplified, giving rise to strongly emitting topologically protected photonic modes.

\section{ACKNOWLEDGMENTS}

We thank O. Bleu for useful discussions. We acknowledge the support of the projects EU "QUANTOPOL" (846353), "Quantum Fluids of Light" (ANR-16-CE30-0021), of the ANR Labex GaNEXT (ANR-11-LABX-0014), and of the ANR program "Investissements d'Avenir" through the IDEX-ISITE initiative 16-IDEX-0001 (CAP 20-25). J.S.M. gratefully acknowledges the project "Hybrid" (ANR-17PIRE-0001). S.V.K. acknowledges the support from the Ministry of Education and Science of the Russian Federation (0791-2020-0006).

\section{APPENDIX A: PROPAGATIVE AND EVANESCENT BOGOLONS}

In the main text, the spectrum of elementary excitations is proven to present a gap. This gap directly comes from the dispersion relation:

$$
E^{2}=\left(\epsilon_{k}+\alpha n-E_{p}\right)\left(\epsilon_{k}+3 \alpha n-E_{p}\right) .
$$

Outside of the gap, for energies $E>\Delta$, this provides two solutions for the square wave vector $k^{2}$ (that is, four solutions for the wave vector $k$ ):

$$
k_{\mp}^{2}=2 m\left(E_{p}-2 \alpha n \pm \sqrt{(\alpha n)^{2}+E^{2}}\right) / \hbar^{2} .
$$



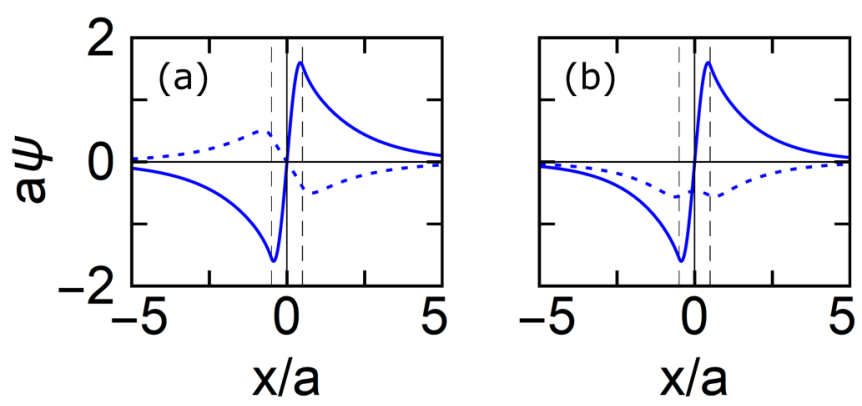

FIG. 5. Andreev particlelike bound states for $a=3 \mu \mathrm{m}$ and for a phase difference of (a) 0 and (b) $\pi / 2$. The dominant (minority) component is shown as a solid (dashed) line. Note the symmetry inversion for the minority component.

However, the solution $k_{+}$is actually imaginary, since the condition $E>\Delta$ exactly corresponds to $E_{p}-2 \alpha n-$ $\sqrt{(\alpha n)^{2}+E^{2}}<0$. This evanescent solution is a continuation of the intragap evanescent "hole"-like state with an inverse decay length $\kappa_{+}$, as shown in Fig. 1(c) of the main text.

The solution $k_{-}$is real, thus leading to propagative states. There are two solutions with the same norm, but opposite propagation direction:

$$
k_{-}= \pm \sqrt{2 m\left(E_{p}-2 \alpha n+\sqrt{(\alpha n)^{2}+E^{2}}\right)} / \hbar .
$$

This is obviously different from the case of evanescent bogolons considered in the main text, where the two inverse decay lengths are different.

For comparison, the energy $E=\hbar \omega$ of a bogolon in the evanescent case can be expressed with respect to the inverse decay length $\kappa$ :

$$
E^{2}=\left(-\epsilon_{\kappa}+\alpha n-E_{p}\right)\left(-\epsilon_{\kappa}+3 \alpha n-E_{p}\right) .
$$

This expression should be compared with Eq. (A1).

Finally, we note that the descriptions of the normal region based on the Schrödinger equation and on the Bogoliubov-de Gennes equations $(\mathrm{BdG})$ with exactly zero interactions are equivalent. While it may seem that the $\mathrm{BdG}$ equations have two solutions with opposite energies, zero interactions mean that the $\mathrm{BdG}$ matrix is already diagonal and the negative energy enters the wave function only with the minus sign. Thus, these two components simply correspond to two waves propagating in opposite directions with the same energy. This energy is positive when measured from the bottom of the band.

\section{APPENDIX B: EVANESCENT STATES IN THE NORMAL REGION}

In the main text, the case $E_{p}>\Delta$ is considered because it leads to propagative states in the normal region for both positive and negative values of $E$, which is the most interesting case. However, the case with $E_{p}<\Delta$ is possible as well. Then, for incident particles with energies $E>E_{p}$, the reflected part at the energy symmetric with respect to the pump detuning is evanescent. We have solved the reflection problem in this case and obtained a nonzero amplitude of the reflected evanescent wave. Our calculations show that SNS junctions with this type of states can exist as well. They present the same global behavior as for the propagative states [see Figs. 5(a) and 5(b) for the two configurations with a different phase], with the wave function in the normal region being a linear combination of hyperbolic functions. However, the bands they form no longer cross. Indeed, the crossing of the bands in the main text occurred when an original state of the potential well formed by the superfluid gaps had the same energy as the bogolon image of another state. This is not possible when the original states are propagative and the images are evanescent, since they are always at the opposite sides of zero (the bottom of the band in the normal region). Thus, the topologically protected self-amplified interface states discussed in the main text cannot be observed for these bands.

\section{APPENDIX C: ANALYTICAL EXPRESSIONS FOR ANDREEV REFLECTION COEFFICIENTS}

In this Appendix, we present explicit expressions for the reflection coefficients for normal and Andreev reflection.

We start by commenting on the limit of vanishing lifetime used for the analytical calculations. A finite $\gamma$ reduces both reflection coefficients. Its effect is stronger for the case of large penetration length $1 / \kappa_{ \pm}$, discussed in the main text. This occurs especially for $E \rightarrow \Delta$. Therefore, the analytical results that we obtain should not be applied for energies close to the edge of the gap and for particularly narrow gaps, $\Delta \rightarrow 0$.

As was mentioned in the main text, both the wave functions and their derivatives have to be continuous at the interface. This imposes

$$
\begin{aligned}
& \frac{1}{\sqrt{v_{1}}}\left[1+r_{N_{p}}\right]\left(\begin{array}{l}
1 \\
0
\end{array}\right)+\frac{r_{A_{p}}}{\sqrt{v_{2}}}\left(\begin{array}{l}
0 \\
1
\end{array}\right) \\
& =\eta_{+}\left(\begin{array}{l}
u_{+} \\
v_{+}
\end{array}\right)+\eta_{-}\left(\begin{array}{l}
u_{-} \\
v_{-}
\end{array}\right), \\
& \frac{i k_{1}}{\sqrt{v_{1}}}\left[1-r_{N_{p}}\right]\left(\begin{array}{l}
1 \\
0
\end{array}\right)-\frac{i k_{2} r_{A_{p}}}{\sqrt{v_{2}}}\left(\begin{array}{l}
0 \\
1
\end{array}\right) \\
& =-\kappa_{+} \eta_{+}\left(\begin{array}{l}
u_{+} \\
v_{+}
\end{array}\right)-\kappa_{-} \eta_{-}\left(\begin{array}{l}
u_{-} \\
v_{-}
\end{array}\right) .
\end{aligned}
$$

Matching the wave functions and their derivatives at the interface gives an analytical expression for the reflection coefficients:

$$
\begin{aligned}
& r_{N_{p}}=\frac{\left(k_{2}+i \kappa_{-}\right)\left(k_{1}-i \kappa_{+}\right) u_{+} v_{-}-\left(k_{1}-i \kappa_{-}\right)\left(k_{2}+i \kappa_{+}\right) u_{-} v_{+}}{\left(k_{2}+i \kappa_{-}\right)\left(k_{1}+i \kappa_{+}\right) u_{+} v_{-}-\left(k_{1}+i \kappa_{-}\right)\left(k_{2}+i \kappa_{+}\right) u_{-} v_{+}} \\
& r_{A_{p}}=\frac{2 i k_{1}\left(\kappa_{+}-\kappa_{-}\right) v_{-} v_{+}}{\left(k_{2}+i \kappa_{-}\right)\left(k_{1}+i \kappa_{+}\right) u_{+} v_{-}-\left(k_{1}+i \kappa_{-}\right)\left(k_{2}+i \kappa_{+}\right) u_{-} v_{+}} \frac{\sqrt{v_{2}}}{\sqrt{v_{1}}}
\end{aligned}
$$


The preceding expressions can be reformulated to make the phase appear explicitly by considering the group velocities $v_{1,2}=\hbar k_{1,2} / m$ and the relation between the Bogoliubov coefficients $u_{+}=-v_{-} e^{2 i \phi}$ and $v_{+}=u_{-} e^{-2 i \phi}$ :

$$
\begin{aligned}
& r_{N_{p}}=\frac{\left(k_{1}-i \kappa_{-}\right)\left(k_{2}+i \kappa_{+}\right)\left|u_{-}\right|^{2}+\left(k_{2}+i \kappa_{-}\right)\left(k_{1}-i \kappa_{+}\right)\left|v_{-}\right|^{2}}{\left(k_{1}+i \kappa_{-}\right)\left(k_{2}+i \kappa_{+}\right)\left|u_{-}\right|^{2}+\left(k_{2}+i \kappa_{-}\right)\left(k_{1}+i \kappa_{+}\right)\left|v_{-}\right|^{2}}, \\
& r_{A_{p}}=\frac{2 i \sqrt{k_{1} k_{2}}\left(\kappa_{+}-\kappa_{-}\right)\left|u_{-}\right|\left|v_{-}\right|}{\left(k_{1}+i \kappa_{-}\right)\left(k_{2}+i \kappa_{+}\right)\left|u_{-}\right|^{2}+\left(k_{2}+i \kappa_{-}\right)\left(k_{1}+i \kappa_{+}\right)\left|v_{-}\right|^{2}} e^{-2 i \phi} .
\end{aligned}
$$

This form makes explicit the role played by $\phi$, and more specifically the change of sign of the Andreev reflection coefficient when $\phi=\pi / 2$. The reflection coefficients for holes are given as $r_{N_{h}}(E)=r_{N_{p}}(-E)$ and $r_{A_{h}}=r_{A_{p}} e^{4 i \phi}=r_{A} e^{2 i \phi}$.

The dependence on the different energies at stake is hidden in the complexity of the formulas. However, for $E \ll E_{p}$, an approximate expression can be given for both coefficients:

$$
\begin{aligned}
& r_{N_{0}}=\frac{E_{p}+\sqrt{\left(\alpha n-E_{p}\right)\left(3 \alpha n-E_{p}\right)}}{E_{p}-\sqrt{\left(\alpha n-E_{p}\right)\left(3 \alpha n-E_{p}\right)}+i \sqrt{E_{p}}\left(\sqrt{\alpha n-E_{p}}+\sqrt{3 \alpha n-E_{p}}\right)}, \\
& r_{A_{0}}=\frac{i \sqrt{E_{p}}\left(\sqrt{\alpha n-E_{p}}-\sqrt{3 \alpha n-E_{p}}\right)}{E_{p}-\sqrt{\left(\alpha n-E_{p}\right)\left(3 \alpha n-E_{p}\right)}+i \sqrt{E_{p}}\left(\sqrt{\alpha n-E_{p}}+\sqrt{3 \alpha n-E_{p}}\right)} .
\end{aligned}
$$

With these expressions, we clearly notice that the three crucial energies to consider are $E_{p}, \alpha n-E_{p}$, and $3 \alpha_{n}-E_{p}$. The maximal value for Andreev reflection coefficients, $\left|r_{A_{0}}\right|^{2}=$ $2 / 3=2\left|r_{N_{0}}\right|^{2}$, is achieved for $\alpha n \rightarrow E_{p}(\Delta \rightarrow 0)$. However, we note that these values are beyond the domain of the validity of the theory, since for $E=\Delta=0$ any finite decay $\gamma$ plays a non-negligible role. For realistic $\Delta,\left|r_{A}\right|^{2}<\left|r_{N}\right|^{2}$.

In the main text, we also consider the case of a SNS junction with a normal region of width $a$. In the derivation of the energy components of a bound state, based on the scattering matrices of the interfaces formed by the reflection coefficients discussed above, we also need the expression of the scattering matrix describing the propagation of the wave in the normal region $S_{N}$. Regardless of the direction of propagation, this matrix reads

$$
S_{N}=\left(\begin{array}{cc}
e^{i k_{1} a} & 0 \\
0 & e^{i k_{2} a}
\end{array}\right)
$$

This matrix, together with the ones describing the reflection processes on both interfaces, allow one to find a condition for the existence of a bound state that takes the form of a cancellation of a determinant (see the main text). This condition is equivalent to

$$
\left|r_{A}\right|^{2} \cos (2 \phi)=\operatorname{Re}\left[r_{N_{h}}^{*}\left(r_{N_{p}} e^{i k_{-} a}-\frac{1}{r_{N_{p}}} e^{-i k_{+} a}\right)\right],
$$

where $k_{ \pm}=k_{1} \pm k_{2}$, which is more compact and makes explicit the reflection coefficients.

\section{APPENDIX D: PROBABILITY CURRENT}

The probability current $J_{ \pm}$of each energy component $\pm E$ can be computed numerically from the expression

$$
J_{+}=v_{1}\left(|A|^{2}-|B|^{2}\right), J_{-}=v_{2}\left(|C|^{2}-|D|^{2}\right) .
$$

Physically, the probability current of each energy component measures the exchange between particles at $+E$ and particles at $-E$. Thus, it follows that

$$
J_{+} \propto r_{A_{h}}^{R}-r_{A_{p}}^{R}, J_{-} \propto r_{A_{p}}^{R}-r_{A_{h}}^{R} .
$$

The currents calculated from (D1) can be plotted on the same graph as $\pm J_{0} \sin (2 \phi)$, where $J_{0}$ (which is phase-independent) is the maximum absolute value of $J_{ \pm}$(see Fig. 6). One can notice that there is an excellent match between $J_{ \pm}$and $\pm J_{0} \sin (2 \phi)$. This can be understood from the expressions of the current (D2), together with the expressions of the reflection coefficients given in Eq. (C3), which yield

$$
J_{ \pm} \propto \pm \sin (2 \phi)
$$

Finally, we retrieve the expression

$$
J_{ \pm}= \pm J_{0}^{\prime} \sin (2 \phi),
$$

where $J_{0}^{\prime}$ has a small phase dependence, contrary to $J_{0}$. This phase dependence comes from the dependence of the energy of the components of a bound state on the phase difference (via the norm of the reflection coefficients). Although the current of each energy component is nonzero, they are opposite and equal in norm, $J_{+}=-J_{-}$. Thus, they compensate for each

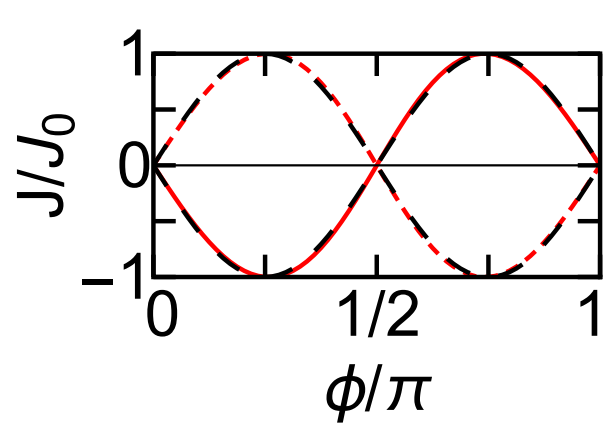

FIG. 6. Numerically calculated probability current of the dominant (red straight line) and minority (red dashed line) energy components for a holelike state. Both follow $\pm \sin (2 \phi)$ behavior (black dashed lines). 
other, and the total current $J_{\text {tot }}$ is null:

$$
J_{\text {tot }}=J_{+}+J_{-}=0 .
$$

\section{APPENDIX E: ZAK PHASE AND BLOCH SPHERE}

To compute the Zak phase, we use equations already introduced in the main text:

$$
\Phi_{\mathrm{Zak}}=\int\left\langle X \mid i \frac{\partial X}{\partial \phi}\right\rangle d \phi
$$

The wave function in the normal (central) region is written as

$$
\psi_{N}=\left(\begin{array}{l}
A e^{i k_{1} x}+B e^{-i k_{1} x} \\
C e^{i k_{2} x}+D e^{-i k_{2} x}
\end{array}\right)
$$

and the associated vectors for each energy component $\pm E$ are written as

$$
X^{+}=\left(\begin{array}{c}
A^{\prime} \\
B^{\prime}
\end{array}\right) \text { and } X^{-}=\left(\begin{array}{c}
C^{\prime} \\
D^{\prime}
\end{array}\right),
$$

where the coefficients are the ones defined in (E2) but normalized to $1\left(\left|A^{\prime}\right|^{2}+\left|B^{\prime}\right|^{2}=1\right.$ and $\left.\left|C^{\prime}\right|^{2}+\left|D^{\prime}\right|^{2}=1\right)$. The coefficients themselves are computed numerically. Such vectors can indeed be plotted on the Bloch sphere. The vector corresponding to the dominant energy always gives $\Phi_{\text {Zak }}=0$, while the other one gives $\Phi_{\mathrm{Zak}}=\pi$.

We note that both spinors are constrained to the great circle of the Bloch sphere by the symmetry of the probability density distribution. Indeed, since the problem is completely symmetric with respect to $x=0$, the probability density has to exhibit mirror symmetry with respect to this point. For this, the relative phase between $A$ and $B$ and also between $C$ and $D$ has to be either 0 or $\pi$, which means that the pseudospin can only make a circle through the constant longitude plane (azimuthal angles $0^{\circ}$ and $180^{\circ}$ ). If one allows an arbitrary phase between these coefficients, the probability density is shifted and becomes asymmetric. Indeed,

$$
A e^{i k_{1} x}+B e^{-i k_{1} x}=(A+B) \cos k_{1} x+i(A-B) \sin k_{1} x .
$$

If the phase difference between $A$ and $B$ is 0 or $\pi$, we can assume that both are real. In this case, the probability density simply reads

$$
\begin{aligned}
& \left|A e^{i k_{1} x}+B e^{-i k_{1} x}\right|^{2} \\
& \quad=(A+B)^{2} \cos ^{2} k_{1} x+(A-B)^{2} \sin ^{2} k_{1} x \\
& \quad=1+2 A B \cos ^{2} k_{1} x,
\end{aligned}
$$

which is symmetric. We can now introduce the phase difference between the two coefficients explicitly:

$$
\begin{aligned}
A e^{i k_{1} x}+B e^{i \varphi} e^{-i k_{1} x}= & e^{i \varphi / 2}\left(A e^{-i \varphi / 2} e^{i k_{1} x}+B e^{i \varphi / 2} e^{-i k_{1} x}\right) \\
= & e^{i \varphi / 2}\left[(A+B) \cos \left(k_{1} x-\varphi / 2\right)\right. \\
& \left.\left.+i(A-B) \sin \left(k_{1} x-\varphi / 2\right)\right], \quad \text { (E } 6\right)
\end{aligned}
$$

which gives an asymmetric probability density

$$
\left|A e^{i k_{1} x}+B e^{i \varphi} e^{-i k_{1} x}\right|^{2}=1+2 A B \cos ^{2}\left(k_{1} x-\varphi / 2\right) .
$$

We conclude that the azimuthal angle on the Bloch sphere $\varphi$ has to be zero, and that the pseudospin has to follow the great circle.

\section{APPENDIX F: NONTOPOLOGICAL CONFIGURATION OF ANDREEV BOUND STATES}

To confirm that the band crossing and amplification observed in the main text are indeed due to the topology of the bands, we consider an alternative configuration where the bands do not exhibit any inversion of the topology with the variation of the parameter, and thus they anticross instead of crossing each other.

We introduce an additional potential barrier at $x=0$, splitting the trap into two parts with two distinct trapped states (left- and right-localized) having the same symmetry ( $s$ for the
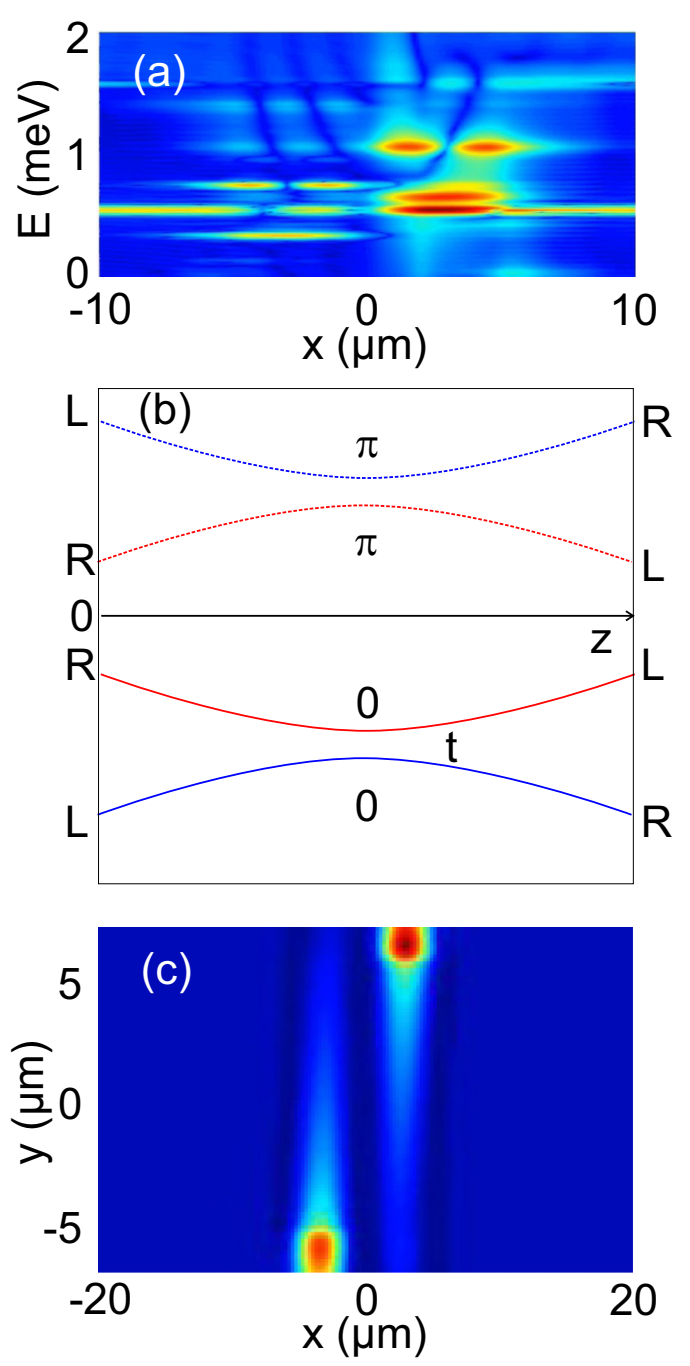

FIG. 7. (a) The intensity of the Andreev states as a function of position and energy for $U_{1} y / l \neq 0$ and $\varphi=\pi / 2$. The two original states are strongly detuned, and their bogolon images are detuned as well. (b) The variation of the energies of the original states and their images as a function of left-right detuning $U_{1} y / l$. The anticrossing is due to the tunneling. The Zak phase indicates that the topology of the bands does not change. (c) The real-space image with the detuning varied along $y$ : no instability is visible at $y=0$. 
lowest state). We also introduce a potential step, responsible for the detuning of these two states. Instead of varying the width of the trap $a$, we vary the height of the step as a function of the second coordinate $y$. The total potential therefore reads

$$
U(x, y)=U_{0} \exp \left(-\frac{x^{2}}{2 \sigma^{2}}\right)+U_{1} \frac{y}{l} \operatorname{sgn}(x)
$$

where $U_{0}=2 \mathrm{meV}$ is the barrier height, $\sigma=0.6 \mu \mathrm{m}$ is its width, $U_{1}=0.2 \mathrm{meV}$ is the characteristic step height, and $l=$ $50 \mu \mathrm{m}$ is the characteristic variation length of the step height.

The results of numerical simulations of this configuration are shown in Fig. 7. Panel (a) presents an example of the calculated spectrum of the Andreev bound states for a particular value of $U_{1} y / l=0.15 \mathrm{meV}$ (the total detuning between the left and right states is $0.3 \mathrm{meV}$ ). The original (s-type) states are clearly visible, as well as their bogolon images exhibiting $p$-symmetry due to the laser phase $\phi=\pi / 2$. Each of the four visible states belongs to a band (as a function of the synthetic variable $\phi$ ). The Zak phases of the bands are calculated as in the main text. They are shown in Fig. 7(b), together with the energies of the band extrema at $\phi=\pi / 2$ plotted as a function of the step height. The two lowest bands, formed from the original $s$-symmetric states, have a zero Zak phase. Their symmetry is the same. Thus, when the step height changes sign and the detuning inversion leads to the state inversion (the lowest state changes localization from left to right), the topology of the system does not change. There are no topological reasons for the crossing of the bands, and indeed, it does not occur: their anticrossing is controlled by the tunneling $t$ across the barrier in the center (controlled by its height $U_{0}$ ). The same concerns the two upper bands, sharing the same topology (Zak phase $\pi$, different from the two lowest bands). Finally, panel (c) confirms that no amplification due to a band crossing occurs in this case (since the crossing is actually avoided): as the step height changes with $y$, the detuning of the states with respect to the laser changes, and we observe the transfer of maximal intensity from left to right, but no signs of macroscopically populated oscillating modes are visible. This confirms that the band crossing and the resulting mode amplification discussed in the main text are indeed of a topological origin.
[1] L. Onsager, Statistical hydrodynamics, Nuovo Cimento 6, 249 (1949).

[2] L. Pitaevskii and S. Stringari, Bose-Einstein Condensation, International Series of Monographs on Physics No. 116 (Oxford Science Publications, Oxford, 2003).

[3] W. P. Su, J. R. Schrieffer, and A. J. Heeger, Solitons in Polyacetylene, Phys. Rev. Lett. 42, 1698 (1979).

[4] D. J. Thouless, M. Kohmoto, M. P. Nightingale, and M. den Nijs, Quantized Hall Conductance in a Two-Dimensional Periodic Potential, Phys. Rev. Lett. 49, 405 (1982).

[5] F. D. M. Haldane, Model for a Quantum Hall Effect without Landau Levels: Condensed-Matter Realization of the "Parity Anomaly," Phys. Rev. Lett. 61, 2015 (1988).

[6] F. D. M. Haldane and S. Raghu, Possible Realization of Directional Optical Waveguides in Photonic Crystals with Broken Time-Reversal Symmetry, Phys. Rev. Lett. 100, 013904 (2008).

[7] Y. Hatsugai, Chern Number and Edge States in the Integer Quantum Hall Effect, Phys. Rev. Lett. 71, 3697 (1993).

[8] R. S. K. Mong and V. Shivamoggi, Edge states and the bulkboundary correspondence in Dirac Hamiltonians, Phys. Rev. B 83, 125109 (2011).

[9] M. Z. Hasan and C. L. Kane, Colloquium: Topological insulators, Rev. Mod. Phys. 82, 3045 (2010).

[10] Y. Lumer, Y. Plotnik, M. C. Rechtsman, and M. Segev, SelfLocalized States in Photonic Topological Insulators, Phys. Rev. Lett. 111, 243905 (2013).

[11] C.-E. Bardyn, T. Karzig, G. Refael, and T. C. H. Liew, Chiral Bogoliubov excitations in nonlinear bosonic systems, Phys. Rev. B 93, 020502(R) (2016).

[12] O. Bleu, D. D. Solnyshkov, and G. Malpuech, Interacting quantum fluid in a polariton Chern insulator, Phys. Rev. B 93, 085438 (2016).

[13] O. Bleu, D. D. Solnyshkov, and G. Malpuech, Photonic versus electronic quantum anomalous Hall effect, Phys. Rev. B 95, 115415 (2017).
[14] D. R. Gulevich, D. Yudin, D. V. Skryabin, I. V. Iorsh, and I. A. Shelykh, Exploring nonlinear topological states of matter with exciton-polaritons: Edge solitons in kagome lattice, Sci. Rep. 7, 1780 (2017).

[15] Y. V. Kartashov and D. V. Skryabin, Bistable Topological Insulator with Exciton-Polaritons, Phys. Rev. Lett. 119, 253904 (2017).

[16] O. Bleu, G. Malpuech, and D. D. Solnyshkov, Robust quantum valley Hall effect for vortices in an interacting bosonic quantum fluid, Nat. Commun. 9, 3991 (2018).

[17] S. Kruk, A. Poddubny, D. Smirnova, L. Wang, A. Slobozhanyuk, A. Shorokhov, I. Kravchenko, B. Luther-Davies, and Y. Kivshar, Nonlinear light generation in topological nanostructures, Nat. Nanotechnol. 14, 126 (2019).

[18] D. Smirnova, D. Leykam, Y. Chong, and Y. Kivshar, Nonlinear topological photonics, Appl. Phys. Rev. 7, 021306 (2020).

[19] D. D. Solnyshkov, A. V. Nalitov, and G. Malpuech, KibbleZurek Mechanism in Topologically Nontrivial Zigzag Chains of Polariton Micropillars, Phys. Rev. Lett. 116, 046402 (2016).

[20] P. St-Jean, V. Goblot, E. Galopin, A. Lemaître, T. Ozawa, L. Le Gratiet, I. Sagnes, J. Bloch, and A. Amo, Lasing in topological edge states of a one-dimensional lattice, Nat. Photon. 11, 651 (2017).

[21] B. Bahari, A. Ndao, F. Vallini, A. El Amili, Y. Fainman, and B. Kanté, Nonreciprocal lasing in topological cavities of arbitrary geometries, Science 358, 636 (2017).

[22] M. A. Bandres, S. Wittek, G. Harari, M. Parto, J. Ren, M. Segev, D. N. Christodoulides, and M. Khajavikhan, Topological insulator laser: Experiments, Science 359, 4005 (2018).

[23] M. Lohse, C. Schweizer, H. M. Price, O. Zilberberg, and I. Bloch, Exploring 4d quantum Hall physics with a 2d topological charge pump, Nature (London) 553, 55 (2018). 
[24] R.-P. Riwar, M. Houzet, J. S. Meyer, and Y. V. Nazarov, Multi-terminal Josephson junctions as topological matter, Nat. Commun. 7, 11167 (2016).

[25] A. W. Draelos, M.-T. Wei, A. Seredinski, H. Li, Y. Mehta, K. Watanabe, T. Taniguchi, I. V. Borzenets, F. Amet, and G. Finkelstein, Supercurrent flow in multiterminal graphene Josephson junctions, Nano Lett. 19, 1039 (2019).

[26] N. Pankratova, H. Lee, R. Kuzmin, K. Wickramasinghe, W. Mayer, J. Yuan, M. G. Vavilov, J. Shabani, and V. E. Manucharyan, Multiterminal Josephson Effect, Phys. Rev. X 10, 031051 (2020).

[27] A. F. Andreev, The thermal conductivity of the intermediate state in superconductors, J. Exp. Theor. Phys. 46, 1823 (1964) [Sov. Phys. JETP 19, 1228 (1964)].

[28] I. Zapata and F. Sols, Andreev Reflection in Bosonic Condensates, Phys. Rev. Lett. 102, 180405 (2009).

[29] I. Carusotto and C. Ciuti, Quantum fluids of light, Rev. Mod. Phys. 85, 299 (2013).

[30] A. Kavokin, J. J. Baumberg, G. Malpuech, and F. P. Laussy, Microcavities (Oxford University Press, Oxford, 2017).

[31] V. Goblot, H. S. Nguyen, I. Carusotto, E. Galopin, A. Lemaître, I. Sagnes, A. Amo, and J. Bloch, Phase-Controlled Bistability of a Dark Soliton Train in a Polariton Fluid, Phys. Rev. Lett. 117, 217401 (2016).

[32] S. V. Koniakhin, O. Bleu, D. D. Stupin, S. Pigeon, A. Maitre, F. Claude, G. Lerario, Q. Glorieux, A. Bramati, D. Solnyshkov, and G. Malpuech, Stationary Quantum Vortex Street in a Driven-Dissipative Quantum Fluid of Light, Phys. Rev. Lett. 123, 215301 (2019).

[33] F. Claude, S. V. Koniakhin, A. Maître, S. Pigeon, G. Lerario, D. D. Stupin, Q. Glorieux, E. Giacobino, D. Solnyshkov, G. Malpuech et al., Taming the snake instabilities in a polariton superfluid, Optica 7, 1660 (2020).

[34] E. Wertz, L. Ferrier, D. Solnyshkov, R. Johne, D. Sanvitto, A. Lemaître, I. Sagnes, R. Grousson, A. V. Kavokin, P. Senellart, G. Malpuech, and J. Bloch, Spontaneous formation and optical manipulation of extended polariton condensates, Nat. Phys. 6, 860 (2010).

[35] L. A. Lugiato and R. Lefever, Spatial Dissipative Structures in Passive Optical Systems, Phys. Rev. Lett. 58, 2209 (1987).

[36] A. Baas, J. P. Karr, H. Eleuch, and E. Giacobino, Optical bistability in semiconductor microcavities, Phys. Rev. A 69, 023809 (2004).

[37] G. E. Blonder, M. Tinkham, and T. M. Klapwijk, Transition from metallic to tunneling regimes in superconducting microconstrictions: Excess current, charge imbalance, and supercurrent conversion, Phys. Rev. B 25, 4515 (1982).

[38] H. Van Houten and C. Beenakker, Andreev reflection and the Josephson effect in a quantum point contact: An analogy with phase-conjugating resonators, Phys. B 175, 187 (1991).

[39] J. C. J. Paasschens, M. J. M. de Jong, P. W. Brouwer, and C. W. J. Beenakker, Reflection of light from a disordered medium backed by a phase-conjugating mirror, Phys. Rev. A 56, 4216 (1997).

[40] A. Yariv, Phase conjugate optics and real-time holography, IEEE J. Quantum Electron. 14, 650 (1978).
[41] C. W. J. Beenakker, Universal Limit of Critical-Current Fluctuations in Mesoscopic Josephson Junctions, Phys. Rev. Lett. 67, 3836 (1991).

[42] N. R. Cooper, J. Dalibard, and I. B. Spielman, Topological bands for ultracold atoms, Rev. Mod. Phys. 91, 015005 (2019).

[43] J. Zak, Berry's Phase for Energy Bands in Solids, Phys. Rev. Lett. 62, 2747 (1989).

[44] P. Delplace, D. Ullmo, and G. Montambaux, Zak phase and the existence of edge states in graphene, Phys. Rev. B 84, 195452 (2011).

[45] H. Shen, B. Zhen, and L. Fu, Topological Band Theory for Non-Hermitian Hamiltonians, Phys. Rev. Lett. 120, 146402 (2018).

[46] B. A. Bernevig, T. L. Hughes, and S.-C. Zhang, Quantum spin Hall effect and topological phase transition in $\mathrm{HgTe}$ quantum wells, Science 314, 1757 (2006).

[47] R. Jackiw and C. Rebbi, Solitons with fermion number 1/2, Phys. Rev. D 13, 3398 (1976).

[48] C. M. Bender and S. Boettcher, Real Spectra in Non-Hermitian Hamiltonians Having Pt Symmetry, Phys. Rev. Lett. 80, 5243 (1998).

[49] Ş. Özdemir, S. Rotter, F. Nori, and L. Yang, Parity-time symmetry and exceptional points in photonics, Nat. Mater. 18, 783 (2019).

[50] G. Cerullo and S. De Silvestri, Ultrafast optical parametric amplifiers, Rev. Sci. Instrum. 74, 1 (2003).

[51] J. H. Eberly, N. B. Narozhny, and J. J. Sanchez-Mondragon, Periodic Spontaneous Collapse and Revival in a Simple Quantum Model, Phys. Rev. Lett. 44, 1323 (1980).

[52] G. Rempe, H. Walther, and N. Klein, Observation of Quantum Collapse and Revival in a One-Atom Maser, Phys. Rev. Lett. 58, 353 (1987).

[53] S. A. Akhmanov, A. I. Kovrigin, A. S. Piskarskas, V. V. Fadeev, and R. V. Khokhlov, Observation of parametric amplification in the optical range, JETP Lett. 2, 191 (1965).

[54] J. A. Giordmaine and R. C. Miller, Tunable Coherent Parametric Oscillation in $\mathrm{Linbo}_{3}$ at Optical Frequencies, Phys. Rev. Lett. 14, 973 (1965).

[55] C. Ciuti, P. Schwendimann, and A. Quattropani, Theory of polariton parametric interactions in semiconductor microcavities, Semicond. Sci. Technol. 18, S279 (2003).

[56] R. Baumgartner and R. Byer, Optical parametric amplification, IEEE J. Quantum Electron. 15, 432 (1979).

[57] J. E. Sharping, K. F. Lee, M. A. Foster, A. C. Turner, B. S. Schmidt, M. Lipson, A. L. Gaeta, and P. Kumar, Generation of correlated photons in nanoscale silicon waveguides, Opt. Express 14, 12388 (2006).

[58] X. Liu, R. M. Osgood, Y. A. Vlasov, and W. M. Green, Midinfrared optical parametric amplifier using silicon nanophotonic waveguides, Nat. Photon. 4, 557 (2010).

[59] A. Schilke, C. Zimmermann, P. W. Courteille, and W. Guerin, Optical parametric oscillation with distributed feedback in cold atoms, Nat. Photon. 6, 101 (2012).

[60] B. I. Sturman, S. Odoulov, and M. Y. Goulkov, Parametric fourwave processes in photorefractive crystals, Phys. Rep. 275, 197 (1996). 